\title{
Coherent Control of Interacting Particles Using Dynamical and Aharonov-Bohm Phases
}

\author{
C. E. Creffield \\ Departamento de Física de Materiales, Universidad Complutense de Madrid, E-28040, Spain \\ G. Platero \\ Instituto de Ciencia de Materiales, CSIC, Cantoblanco, Madrid, E-28049, Spain \\ (Received 1 June 2010; published 19 August 2010)
}

\begin{abstract}
A powerful method of manipulating the dynamics of quantum coherent particles is to control the phase of their tunneling. We consider a system of two electrons hopping on a quasi-one-dimensional lattice in the presence of a uniform magnetic field and study the effect of adding a time-periodic driving potential. We show that the dynamical phases produced by the driving can combine with the Aharonov-Bohm phases to give precise control of the localization and dynamics of the particles, even in the presence of strong particle interactions.
\end{abstract}

DOI: 10.1103/PhysRevLett.105.086804

PACS numbers: 73.23.- b, 03.65.Xp, 03.67.Bg

Introduction.-Experimental advances in producing low-dimensional semiconductor nanostructures have given us the opportunity of studying quantum transport in regimes ranging from noninteracting to strongly correlated. The excellent coherence properties of these devices, together with the degree of control over their geometry and specifications, make them ideal candidates for studying coherent transport, where quantum interference is used to regulate the movement of particles. Such control is particularly vital for quantum information applications, in which the coherence and entanglement of the initial state must be preserved during the evolution of the system.

If we consider a particle hopping on a lattice, interference will occur if the hopping acquires a phase factor. A direct way of doing this is to apply a magnetic field, which produces the well-known Aharonov-Bohm (AB) phase. In Ref. [1] it was shown that such phases could produce a localization effect termed $A B$ caging, in which destructive interference bounds the set of sites that can be visited by an initially localized wave packet. For example, in the rhombus-chain lattice shown in Fig. 1(a), a particle initialized on the central site will not spread out along the lattice if each plaquette is threaded by a single flux quantum $(\Phi=$ $\pi)$. This caging effect has been observed experimentally in superconducting wire networks [2], mesoscopic semiconductor lattices [3], and arrays of Josephson junctions [4].

A different form of localization, also arising from quantum interference, is termed [5] "coherent destruction of tunneling" (CDT). This arises in systems subjected to a time-periodic driving field. Tunneling processes acquire phase factors from the interaction of the system with the driving, producing an effective renormalization of the tunneling. For the most common case of sinusoidal driving, for example, the single-particle tunneling is renormalized by the zeroth Bessel function $\mathcal{J}_{0}$ [6].

$\mathrm{AB}$ caging is resistant to small quantities of disorder [7] but is swiftly destroyed by interactions [see Fig. 1(b)] due to the formation of spatially extended states [8]. In this Letter, we consider a system of two interacting electrons and show that, by combining a high-frequency driving potential with the magnetic flux, stable $\mathrm{AB}$ caging can be restored. This occurs when CDT causes the (repulsive) electrons to bind together into a composite object of charge $2 e$ termed a "doublon," which can then be caged by the magnetic flux. We then go on to consider the effect of a low-frequency driving field and show that this gives rise to an unusual form of propagation in which the doublon

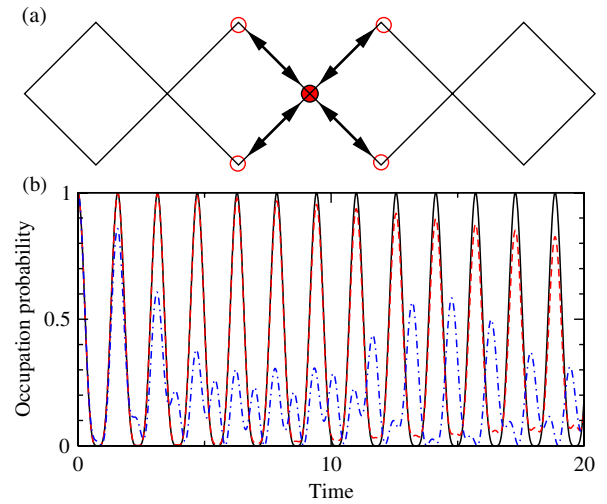

FIG. 1 (color online). (a) Schematic arrangement of the "rhombus-chain" lattice. When each plaquette is threaded by a flux $\Phi=\pi$, a particle initialized on the central site (filled circle) will oscillate to its nearest neighbors (empty circles) but will not propagate through the lattice. The circled sites form an Aharonov-Bohm cage. (b) Occupation probability of the central site for a two-electron system. The noninteracting case (solid black line) displays regular, nondecaying oscillations, indicating perfect $\mathrm{AB}$ caging. For weak interaction $(U=0.1 \mathrm{~J}$, dashed red line) the oscillations decay; the caging is no longer complete, and the particle can leak away. For stronger interactions $(U=J$, dot-dashed blue line) the oscillations decay even more rapidly and soon become irregular. 
moves in steps of two lattice sites, via the virtual occupation of the intermediate sites.

Model.-We consider a system of two electrons hopping on a chain of connected rhombi, as shown in Fig. 1(a). When propagation from site to site is coherent, this system can be modeled well [9] by a single-band Hubbard model

$$
H_{0}=-J \sum_{\langle j, k\rangle \sigma}\left[e^{i \phi_{j k}} c_{j \sigma}^{\dagger} c_{k \sigma}+\text { H.c. }\right]+U \sum_{j} n_{j\rceil} n_{j \downarrow},
$$

where $J$ is the intersite tunneling between nearest neighbors $\langle j, k\rangle$ and $U$ represents the energy cost of doubly occupying a lattice site. The operators $c_{j \sigma}^{\dagger}\left(c_{j \sigma}\right)$ are the usual creation (annihilation) operators for an electron of spin $\sigma$ on site $j$, and $n_{j \sigma}$ is the standard number operator. Each rhombus is threaded by a magnetic flux $\Phi$, giving rise to the $\mathrm{AB}$ phases $\exp \left[i \phi_{j k}\right]$ on the tunneling terms. In semiconductor quantum dots, singlet-triplet mixing terms are typically rather weak [10], and accordingly we neglect their effect here. We also confine our attention in this work to the singlet subspace, where one electron is spin-up and the other is spin-down, since in the triplet subspace the Hubbard interaction is not operative.

Results.-In Fig. 1(b), we show the time evolution of the system's wave function by plotting its overlap with the initial state, which consists of both electrons occupying the central site. In order to produce $\mathrm{AB}$ caging we set the applied flux to $\Phi=\pi$ [1]. In the absence of interactions $(U=0)$, the overlap displays regular sinusoidal oscillations, indicating that the pair of electrons periodically reverts to their original configuration. Examining their dynamics in detail reveals that the electrons periodically oscillate from the initial site to its nearest neighbors but propagate no further down the lattice due to the caging effect. Raising the interaction strength to $U=0.1 J$ causes the $\mathrm{AB}$ cage to partially open, allowing the electrons to spread over the whole lattice. As $U$ is increased further, this leakage occurs more rapidly.

We now consider adding a periodically oscillating potential that rises linearly along the lattice:

$$
H(t)=H_{0}+E \sin \omega t \sum_{j} x_{j}\left(n_{j \uparrow}+n_{j \downarrow}\right) .
$$

Here $E$ and $\omega$ parameterize the amplitude and frequency of the potential, respectively, and $x_{j}$ is the $x$ component of the location of site $j$. As $H(t)$ is periodic in time, $H(t)=$ $H(t+T)$, where $T=2 \pi / \omega$ is the period of the driving, the Floquet theorem allows us to write solutions of the Schrödinger equation in the form $|\Psi(t)\rangle=e^{-i \epsilon_{n} t}\left|\psi_{n}(t)\right\rangle$. Here $\epsilon_{n}$ are the Floquet quasienergies, and $\left|\psi_{n}(t)\right\rangle$ are a set of $T$-periodic functions termed Floquet states. The quasienergies represent the appropriate generalization of energy eigenvalues to the case of a periodically driven system and have an analogous role in elucidating the system's behavior. In particular, exact or near degeneracy of quasienergies results in the suppression of tunneling between the associated Floquet states: CDT [5].

High frequency $(\omega>U)$. - In Fig. 2(a), we show the Floquet quasienergies, obtained [9] by the exact diagonalization of the one-period propagator of the many-body Hamiltonian (2), for a system in the high-frequency regime. We see that the quasienergies fall into two minibands: the higher miniband corresponding to Floquet states in which sites are doubly occupied, and the lower to states in which the electrons are separated in space. Both bands "collapse" at specific values of $E / \omega$. In the highfrequency regime, a perturbative calculation of the Floquet system $[9,11]$ reveals that the driving potential has the effect of renormalizing the tunneling to an effective value $J \rightarrow J_{\text {eff }}=J \mathcal{J}_{0}(E x / \omega)$, where $x=1 / 2$ is the $x$ component of the spacing between neighboring lattice sites in units of the lattice spacing. Thus when $E / \omega \simeq$ $4.80,11.04 \ldots$, the zeros of $\mathcal{J}_{0}$, the effective tunneling is suppressed and the miniband collapses.

The driving field thus provides a handle to directly control the ratio $U / J_{\text {eff }}$, which can be enhanced by tuning the driving parameters near to a zero of $\mathcal{J}_{0}$. This has been used, for example, to induce the Mott transition in cold
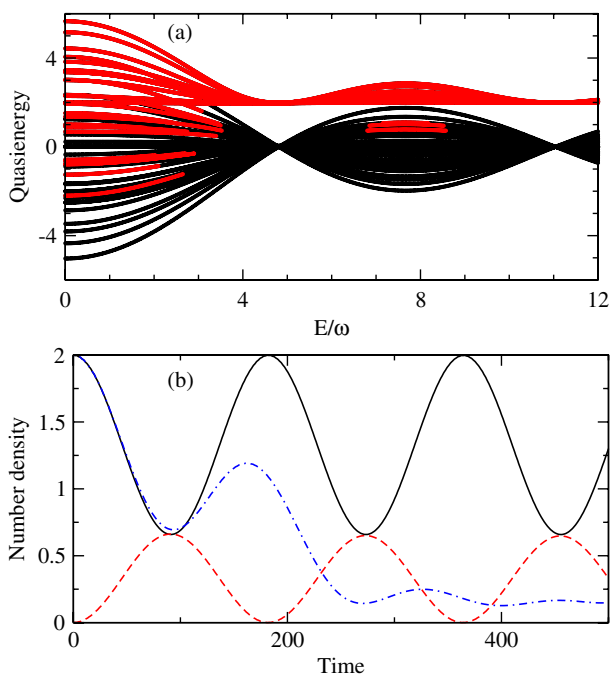

FIG. 2 (color online). (a) Floquet quasienergies for a periodically driven two-electron system. Parameters are $U=2 J$ and $\omega=16 J$ (the high-frequency regime). The quasienergies fall into two minibands: The lower (black) corresponds to Floquet states in which the electrons are separated, the higher (red) to states in which both electrons occupy the same site. The minibands are separated by the Hubbard gap $U$. Both minibands collapse at $E / \omega=4.80,11.04$, corresponding to CDT. (b) For $E / \omega=4.80$ and $\Phi=\pi / 2$ the occupation of the central site (solid black line) oscillates as electrons periodically tunnel to and from their nearest neighbors [see Fig. 1(a)]. For ease of comparison, the occupation of the neighboring site (dashed red line) is multiplied by 2 . When $\Phi$ is tuned away from this value, $\mathrm{AB}$ caging does not occur; the dot-dashed blue line shows the occupation of the central site for $\Phi=0$ for which the electrons diffuse throughout the lattice. 
atom systems [12]. Another consequence of enhancing this ratio is the creation of repulsively bound pairs [13,14], or doublons. Since binding is usually associated with attractive forces, it may seem counterintuitive for strong repulsive interactions to also produce this effect. Qualitatively, it can be understood from an energetics argument. The energies of a one-dimensional lattice form a Bloch band with a width of $2 J$, and thus the maximum kinetic energy carried by two free particles is $4 J$. If the particles are initially prepared in a state with a potential energy much greater than $4 J$, the initial state then cannot decay without the mediation of dissipative processes. In sufficiently clean systems the only decay path [15] is from scattering with unbound particles, resulting in a doublon lifetime that depends exponentially on $U / J_{\text {eff }}$.

To observe the dynamics of doublons we require $J_{\text {eff }}$ to be close to zero (to enhance the ratio $U / J_{\text {eff }}$ for doublon formation to occur) but to be sufficiently large for the doublon tunneling to be non-negligible. In Fig. 2(b), we show the occupation of the central site and its nearest neighbors for driving parameters $E / \omega=4.80$. For general values of the magnetic flux $\Phi$, the doublon slowly diffuses through the lattice, the two electrons remaining bound together but not being localized. For the case of $\Phi=$ $\pi / 2$, however, the dynamics again shows a regular oscillatory motion due to $\mathrm{AB}$ caging. Thus, even though the system is strongly interacting $(U=2 J), \mathrm{AB}$ caging can nonetheless be induced. Since the doublon has a charge of $2 e$, however, the caging now occurs when $\Phi$ is equal to half a flux quantum.

Resonant frequency.-We now consider raising the interaction strength further. When $\omega$ is no longer the dominant energy scale, the simple perturbation theory must be generalized to include interactions $[9,16]$. This reveals that in this regime the effect of the driving field is particularly strong when it is resonant with the driving frequency $U=$ $n \omega$, where $n$ is an integer.

In Fig. 3(a), we show the quasienergies obtained for parameters $U=16 \mathrm{~J}$ and $\omega=16 \mathrm{~J}$ : the $n=1$ resonance. As before, the Floquet states in which the electrons are separated fall into a miniband modulated by $\mathcal{J}_{0}(E / 2 \omega)$. As predicted by perturbation theory [13], however, the quasienergies in which the Hubbard interaction is operative are instead modulated by $\mathcal{J}_{n}(E / 2 \omega)$, where $n$ is the order of the resonance. Consequently, doublon formation now occurs when $E / 2 \omega$ is tuned near to a zero of $\mathcal{J}_{1}$. This is shown in Fig. 3(b); when $E / \omega=7.65$, close to the first zero of $\mathcal{J}_{1}$ a stable doublon is formed, which undergoes $\mathrm{AB}$ caging when the applied flux is set to $\Phi=\pi / 2$. As before, $A B$ caging occurs only for this specific flux value, and for other values of $\Phi$ the doublon simply spreads through the lattice. It is interesting to note that, unlike the high-frequency case, the oscillation is now complete; the central site empties completely as the doublon tunnels to the other sites in the $\mathrm{AB}$ cage. This is a consequence of
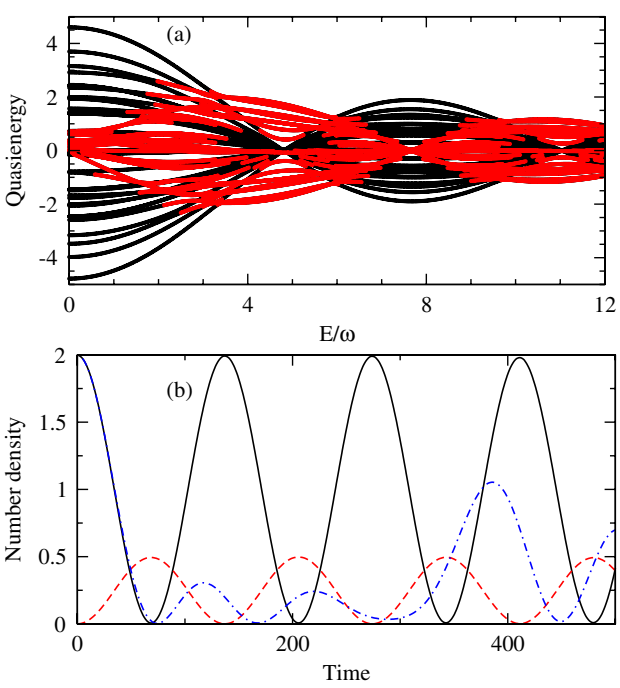

FIG. 3 (color online). (a) Resonant driving, $U=16 \mathrm{~J}, \omega=$ $16 J$. Quasienergies corresponding to states in which the electrons are separated (black) are modulated by the zeroth Bessel function $\mathcal{J}_{0}(E / 2 \omega)$. The quasienergies corresponding to doubly occupied states (red) are instead modulated by $\mathcal{J}_{1}(E / 2 \omega)$ and show band collapses at $E / \omega \simeq 7.66,15.44$. (b) For $E / \omega=7.65$ and $\Phi=\pi / 2 \mathrm{AB}$ caging occurs, and the occupation of the central site (solid black line) oscillates from 2 to zero, while correspondingly the occupation of the neighboring sites (dashed red line) oscillates between zero and 0.5. Tuning $\Phi$ away from this value destroys the $\mathrm{AB}$ caging; the dot-dashed blue line shows the occupation of the central site for $\Phi=0$.

the resonance condition. In an effect analogous to photonassisted tunneling, the doublon can absorb energy from the driving field to exactly compensate for $U$, meaning that the system becomes effectively noninteracting.

Low frequency $(\omega<U)$. - Finally, we consider the case when the driving frequency is much smaller than the interaction strength. If $U / J$ is sufficiently large, then doublons will form in the static system, and the driving field can now be used to control their motion. In Fig. 4(a), we show the Floquet spectrum for states in which sites are doubly occupied, which we can regard as a miniband of doublon states. This miniband is clearly modulated by $\mathcal{J}_{0}(2 E x / \omega)$, where the factor of 2 in the argument of the Bessel function occurs because the doublon has charge $2 e$. The miniband structure persists until $E / \omega \geq 8$, which marks the onset of resonant driving [13]. For these parameters this will be an $n=8$ resonance (since $U / \omega=8$ ), but, due to the strong driving potential required to reach this regime, we do not study it further here.

Tuning $E / \omega$ close to 2.40 thus has the effect of suppressing direct tunneling of the doublon. Motion is still possible, however, via second-order tunneling processes; the bound electrons hop to intermediate lattice sites (incurring an energy cost of $U$ ) and then recombine after another hopping process, as illustrated schematically in Fig. 4(c). This motion has the unusual feature that it occurs in steps of two lattice sites, with the intermediate sites being occupied 


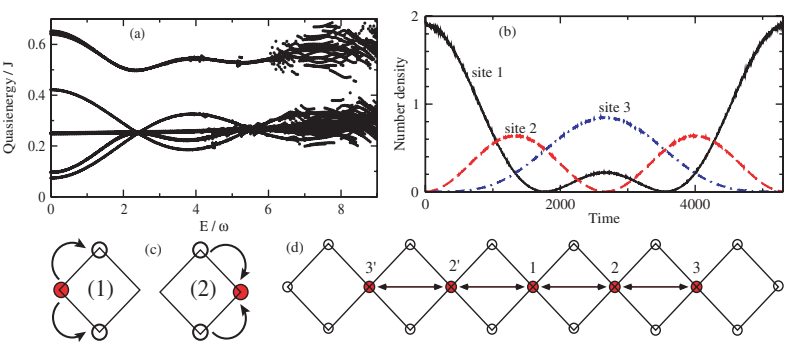

FIG. 4 (color online). (a) For low driving frequencies, $U=$ $16 J, \omega=2 J$, the quasienergies corresponding to doubly occupied states form a miniband modulated by $\mathcal{J}_{0}(E / \omega)$. (b) Doublon dynamics for a 19-site system, where $E / \omega=$ 2.30 to suppress the direct doublon tunneling. The doublon is initialized on the central site 1 (see below) and propagates along the spine of the lattice. After reaching site $3\left(3^{\prime}\right)$ it is reflected and returns to the center. (c) When the doublon tunneling is suppressed, motion occurs via second-order tunneling; the doublon first unbinds (1) and then recombines (2). The doublon thus propagates in steps of two lattice sites, occupying the intermediate sites only virtually. (d) Schematic arrangement of the 19-site lattice. Filled circles indicate spinal sites, empty circles the edge sites. The second-order tunneling clearly does not connect spinal sites to edge sites.

only virtually. If we separate the rhombus-chain lattice into "edge" and "spinal" sites, as shown in Fig. 4(d), it can be easily seen that this tunneling process does not connect spinal sites to edge sites and so produces a separation between these two sublattices.

In Fig. 4(b), we show the result of initializing the system in a doublon state at the center of a 19-site lattice. We can see that the doublon propagates smoothly along the spine of the lattice, the occupation of edge sites always remaining less than 0.006 . This propagation occurs symmetrically to the left and right and so behaves as an electronic beam splitter [17]. On reaching the final sites [sites 3 and $3^{\prime}$, as shown in Fig. 4(d)], the wave packet is reflected and revives in the central site with excellent fidelity. We note that the doublon does not reach site $4\left(4^{\prime}\right)$, although in principle the second-order tunneling process should permit this. This is a finite-size effect [11]; the states that project onto the terminating sites $\left(4\right.$ and $\left.4^{\prime}\right)$ have a different symmetry to the other spinal states and so lie in a different miniband, thereby isolating them from the dynamics of the spinal states.

Conclusions.-Combining $\mathrm{AB}$ phases with a timedependent driving potential gives an extremely rich behavior. Promising systems to observe these effects experimentally are gated semiconductor nanostructures, which have the excellent coherence properties [18] and addressability required. Other interesting possibilities include arrays of coupled Josephson junctions-in which AB caging has already been demonstrated [4] — or cold atoms held in optical lattice potentials $[12,14,15]$. In particular, we have shown that $A B$ trapping can occur in an interacting system by using CDT to convert pairs of electrons into doublons. The resonant behavior displayed by the driven system also provides a convenient means to measure the strength of interactions by suitably tuning the value of $\omega$. Reducing the frequency of the driving gives complete coherent control over the dynamics of the doublons, allowing them to be localized within an $\mathrm{AB}$ cage or to propagate via an unusual second-order tunneling process. This permits the creation and control of spatially separated entangled states of two electrons via the beam-splitter effect, with many potential applications to quantum information.

This research was supported by the Ramón y Cajal program (C.E.C.) and by Spanish MICINN through Grants No. FIS-2007-65723 (C.E.C.) and No. MAT2008-02626 (G. P.).

[1] J. Vidal, R. Mosseri, and B. Douçot, Phys. Rev. Lett. 81, 5888 (1998).

[2] C.C. Abilio, P. Butaud, Th. Fournier, B. Pannetier, J. Vidal, S. Tedesco, and B. Dalzotto, Phys. Rev. Lett. 83, 5102 (1999); B. Pannetier, C. C. Abilio, E. Serret, T. Fournier, P. Butaud, and J. Vidal, Physica (Amsterdam) 352C, 41 (2001).

[3] C. Naud, G. Faini, and D. Mailly, Phys. Rev. Lett. 86, 5104 (2001).

[4] I. M. Pop et al., Phys. Rev. B 78, 104504 (2008); S. Gladchenko et al., Nature Phys. 5, 48 (2009).

[5] F. Grossmann, T. Dittrich, P. Jung, and P. Hänggi, Phys. Rev. Lett. 67, 516 (1991).

[6] D. H. Dunlap and V. M. Kenkre, Phys. Rev. B 34, 3625 (1986); J. H. Shirley, Phys. Rev. 138, B979 (1965).

[7] J. Vidal, P. Butaud, B. Douçot, and R. Mosseri, Phys. Rev. B 64, 155306 (2001).

[8] J. Vidal, B. Douçot, R. Mosseri, and P. Butaud, Phys. Rev. Lett. 85, 3906 (2000).

[9] C.E. Creffield and G. Platero, Phys. Rev. B 65, 113304 (2002).

[10] I. A. Merkulov, Al. L. Efros, and M. Rosen, Phys. Rev. B 65, 205309 (2002).

[11] M. Holthaus, Phys. Rev. Lett. 69, 351 (1992).

[12] A. Zenesini, H. Lignier, D. Ciampini, O. Morsch, and E. Arimondo, Phys. Rev. Lett. 102, 100403 (2009).

[13] C. E. Creffield and G. Platero, Phys. Rev. B 69, 165312 (2004).

[14] K. Winkler et al., Nature (London) 441, 853 (2006).

[15] N. Strohmaier et al., Phys. Rev. Lett. 104, 080401 (2010).

[16] C. E. Creffield and G. Platero, Phys. Rev. B 66, 235303 (2002).

[17] L. Hofstetter, S. Csonka, J. Nygård, and C. Schönenberger, Nature (London) 461, 960 (2009).

[18] G. Shinkai, T. Hayashi, T. Ota, and T. Fujisawa, Phys. Rev. Lett. 103, 056802 (2009). 